\section{Monitoring retinal function in early age-related maculopathy: visual performance after 1 year}

B Feigl ${ }^{1,2}$, B Brown 2 , J Lovie-Kitchin² and P Swann ${ }^{2}$

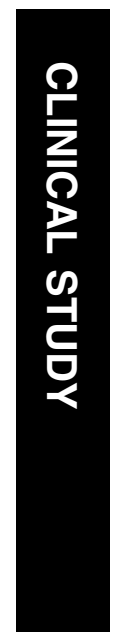

at baseline no further deterioration was

evident after 1 year.

Eye (2005) 19, 1169-1177. doi:10.1038/sj.eye.6701711;

published online 24 September 2004

Keywords: multifocal electroretinogram; early

age-related maculopathy; cone-mediated

function; rod-mediated function; mfERG;

monitoring

\section{Introduction}

Many aspects of retinal function can be altered in early ARM. ${ }^{1-11}$ There have been several studies showing impairment of the photopic and scotopic pathways in early ARM subjects. ${ }^{8,10,12-16}$ Phipps et al ${ }^{12}$ demonstrated an alteration of cone adaptational kinetics by measuring the post-bleaching recovery time to various contrast multiples. Falsini et $a l^{13}$ showed altered temporal cone flicker sensitivity using the focal ERG and could discriminate between different degrees of ARM based upon funduscopic changes. Gerth et $a l^{14}$ and Li et $a l^{15}$ tested cone-mediated function with the multifocal electroretinogram (mfERG) in early ARM and found the latencies to be very sensitive for objective detection of functional impairment. Scholl et $a l^{16}$ showed that retinal areas of increased fundus autofluorescence corresponding to drusen had a greater loss of scotopic rather than photopic sensitivity. Whether cone- or rod-mediated function is first affected still needs to be investigated but there is histopathological ${ }^{9}$ and psychophysical evidence $^{8,17}$ of a preferential rod vulnerability in early ARM. Curcio et $a l^{9,18}$ demonstrated a parafoveal rod loss but a normal foveal cone mosaic and Owsley et al ${ }^{19}$ showed delayed rod-mediated dark adaptation in early ARM.
${ }^{1}$ Department of Ophthalmology University of Graz Austria

${ }^{2}$ Centre for Health Research School of Optometry Queensland University of Technology, Australia

Correspondence: B Feigl Queensland University of Technology School of Optometry Victoria Park Road Australia Tel.: +61738645732 Fax: + 61738645665 E-mail: b.feigi@qut.edu.au

Received: 20 May 2004 Accepted in revised form: 22 July 2004

Published online: 24 September 2004 Kelvin Grove, Q 4059 
Owsley et $a l^{8}$ further showed that mean scotopic sensitivity loss exceeded the magnitude of photopic sensitivity loss in $87 \%$ of their ARM subjects. Hood et al ${ }^{20}$ developed guidelines for eliciting a rod-mediated mfERG and applied it successfully in subjects with hereditary diseases. We adapted a similar protocol to our laboratory conditions and subjects, to show a significant delay in the mean rod-mediated responses in early ARM compared to a control group, whereas the mean cone-mediated responses were within the normal range. ${ }^{21}$

The aim of this study was to assess and monitor coneand rod-mediated function with subjective and objective vision tests in early ARM at baseline and after 1 year. Our hypothesis was that, given the evidence of preferential rod vulnerability, the best indicator of the vision function tests we used for detecting impairment and/or progression of early ARM was the rod-mediated mfERG.

\section{Methods}

\section{Subjects}

From 33 subjects who were initially selected from the Optometry Clinic at the Queensland University of Technology (QUT), Brisbane or were referred by local ophthalmologists and seen at baseline, 26 subjects were followed up after 1 year. Of these, 13 subjects made up the early ARM group (seven female, six male, mean age 72 years) and 13 subjects comprised the age-matched control group (eight females and five males, mean age 70 years). Of the other seven subjects seen at baseline, two developed a chorioretinal neovascularisation $(\mathrm{CNV})$ in the tested eye, four were not able to return for private reasons and one subject had died. All subjects had a distance visual acuity of $6 / 12$ or better and were phakic at both visits. They underwent a full clinical examination, including slitlamp and fundus photography. All subjects who were enrolled in the study gave written informed consent and the tenets of the Declaration of Helsinki and the requirements of the University Human Research Ethics Committee at QUT were followed.

Slitlamp photographs were taken for lens ${ }^{22}$ and fundus grading $^{23}$ which were performed according to the templates of the Age-Related Eye Disease Study (AREDS) as previously described. ${ }^{21}$ Retinal changes were graded independently by two experienced observers (BF, PS) using a set of the Wisconsin age-related maculopathy standards with example photographs and a grid with grading circles (AREDS Reading Center, University of Wisconsin, Madison, WI, USA). ${ }^{23}$ One observer (PS) was masked to the subjects' functional results and agreement was achieved in all subjects with judgments of the other investigator (BF, not masked).

We defined early ARM as the presence of either hard or soft distinct and indistinct drusen greater than $63 \mu \mathrm{m}$ in size with or without retinal pigment epithelial (RPE) abnormalities. Both types of drusen have been shown to damage cone and rod inner and outer segments. ${ }^{24}$ Table 1 shows the characteristics of each ARM subject and the AREDS fundus grading ${ }^{23}$ results at baseline and after 1 year.

\section{mfERGs}

The mfERGs (VERIS I, EDI Inc, San Mateo, CA, USA) were recorded under the same conditions as recently described. ${ }^{21}$ We recorded monocularly by using DTL thread electrodes and optical correction for the stimulus viewing distance $(50 \mathrm{~cm})$. Pupils were maximally dilated

Table 1 Subject characteristics with visual acuities (VA), desat. Panel D-15 error indices (C-index, the higher the index the poorer the colour vision) and type of deficiency in brackets and fundus grading results at baseline and after 1 year

\begin{tabular}{lllllllll}
\hline Subjects & $\begin{array}{c}\text { Age at } \\
\text { first visit }\end{array}$ & Eye & VA baseline & VA 1 year & C-index baseline & C-index 1 year & $\begin{array}{l}\text { Fundus } \\
\text { grading } \\
\text { basline }\end{array}$ & Fundus grading 1 year \\
& & & & & & & & \\
$\mathrm{a}$ & 63 & re & $6 / 6$ & $6 / 7.5^{-1}$ & 1.12 (normal) & 1.15 (normal) & LIIa & L IIa \\
$\mathrm{b}$ & 72 & re & $6 / 12$ & $6 / 6^{-1}$ & 3.14 (Tritan) & 2.94 (Tritan) & L IIIb & LIIIb \\
$\mathrm{c}$ & 73 & le & $6 / 6^{-1}$ & $6 / 9.5^{-1}$ & 1.57 (normal) & 1.22 (normal) & LIIc (3) & LIIc (3) (more pigment) \\
$\mathrm{d}$ & 72 & re & $6 / 12$ & $6 / 9.5^{-1}$ & 2.61 (Tritan) & 2.76 (Tritan) & LIIc (3) & LIIc (3) \\
$\mathrm{e}$ & 73 & re & $6 / 6^{-1}$ & $6 / 9.5^{-2}$ & 1.77 (Tritan) & 2.72 (Tritan) & LIIc (1) & LIIc (1) \\
$\mathrm{f}$ & 69 & le & $6 / 7.5^{-1}$ & $6 / 9^{+2}$ & 1.51 (Tritan) & 1.34 (Tritan) & L IIIb & L IIIb \\
$\mathrm{g}$ & 71 & le & $6 / 7.5^{-2}$ & $6 / 9^{+2}$ & 2.93 (Tritan) & 3.15 (Tritan) & LIIc (3) & LIIc (3) \\
$\mathrm{h}$ & 73 & re & $6 / 7.5$ & $6 / 7.5^{+2}$ & 1.7 (Tritan) & 2.07 (Tritan) & L IIb & L IIb \\
$\mathrm{i}$ & 77 & re & $6 / 7.5$ & $6 / 7.5^{-2}$ & 1.73 (Tritan) & 2.78 (Tritan) & L IIIb & L IIIb \\
$\mathrm{k}$ & 67 & re & $6 / 9.5^{+1}$ & $6 / 12^{+1}$ & 3.22 (Tritan) & 3.61 (Tritan) & L IIIb & LIIc (3) (new pigment) \\
1 & 73 & re & $6 / 9.5^{+1}$ & $6 / 9.5^{+1}$ & Not possible & Not possible & L IIIb & LIIIb \\
$\mathrm{o}$ & 77 & re & $6 / 9.5$ & $6 / 9.5^{-2}$ & 2.92 (nonpolar) & 2.39 (Tritan) & LIIc (3) & LIIc (3) \\
$\mathrm{p}$ & 72 & re & $6 / 6^{-1}$ & $6 / 9.5^{-1}$ & 2.25 (Tritan) & 3.02 (Tritan) & LIIc (3) & LIIc (3) \\
\hline
\end{tabular}


(tropicamide $0.5 \%$ and phenylephrine $2.5 \%$ ) and the subjects were instructed to watch the centre of the monitor. The frame rate of the hexagonal display was $67 \mathrm{~Hz}$, and the hexagons flickered according to a pseudorandom binary $m$-sequence $\left(2^{13}-1\right.$ steps in length). The stimuli for the cones and rods were a 103 $(35.5 \times 28 \mathrm{deg})$ and a 61 hexagon array $(33 \times 28 \mathrm{deg})$, respectively. Retinal signals were bandpass filtered (1-300 Hz) and amplified (Grass P5 amplifier, × 100000$)$. Blinks or small eye movements causing ERG artefacts during the recording segments were detected online and those segments were rejected and re-recorded. We used a cross extending to each corner of the screen to help subjects maintain fixation and controlled fixation by observing the subjects' recordings online on a monitor and by analysing the region of the blind spot after the recordings. Kernel overlap was excluded by performing an overlap test for each protocol.

\section{Cone-mediated mfERG}

The luminance was $100 \mathrm{~cd} / \mathrm{m}^{2}$ for the white hexagons and $2 \mathrm{~cd} / \mathrm{m}^{2}$ for the black hexagons (all our luminance measures were made with a Topcon BM-7 luminancecolorimeter). We divided the recordings into 16 segments (each about $11 \mathrm{~s}$ ) and four files for averaging were obtained from every subject (total recording time was about $10 \mathrm{~min}$ per eye, not including resting time between the segments).

\section{Rod-mediated mfERG}

Rod-mediated mfERGs were recorded after 40 min dark adaptation using our protocol ${ }^{21}$ (61 unscaled hexagons, Wratten 47B with ND filters and slowed stimulus sequence by inserting three blank frames) following the recommendations of Hood et al. ${ }^{20}$ The luminance levels were $0.0098 \mathrm{~cd} / \mathrm{m}^{2}$ for the bright, $0.0005 \mathrm{~cd} / \mathrm{m}^{2}$ for the dark hexagons, and $0.004 \mathrm{~cd} / \mathrm{m}^{2}$ for the surround. Figure 1a and $b$ shows hexagonal trace arrays for the cone- (a) and rod-mediated (b) results with the averaged local responses of the age-matched control group. The superimposed waveforms indicate the overall responses. The rod-mediated responses are smaller and broader than those of the cones with late, less distinct peaks.

\section{Analysis}

We applied Hood and Li's ${ }^{25}$ computerized fitting method $^{26-30}$ and derived normal templates for each of the 103 and 61 hexagons by averaging the local mfERGs recorded from the 13 control subjects at baseline. We then compared the control and ARM subjects' baseline and 1 year amplitude (a-scale) and latency ( $t$-scale) results
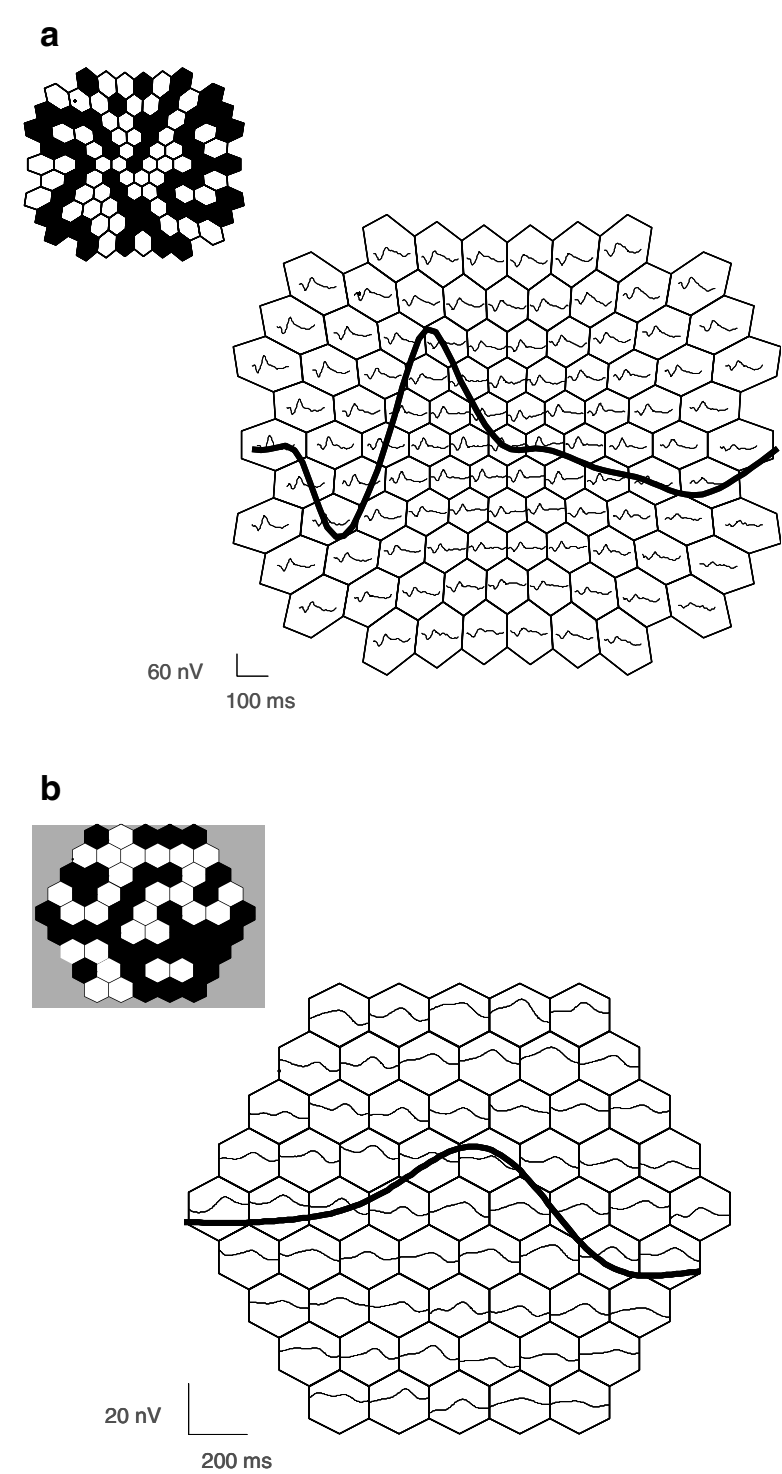

Figure 1 The averaged (a) cone- and (b) rod-mediated mfERG responses of the age-matched control group with the hexagonal stimulus arrays above. The superimposed waveforms indicate the overall response. Note the different waveform shapes, with smaller and later rod-mediated responses without a distinct peak.

against those templates. The quality of fit cutoff was set at $0.8^{21,25-27,29,30}$ and results higher than 0.8 were considered as noise. The records of the left eyes were mirror-imaged so that appropriate parts of the retina were being compared across eyes.

Before fitting the cone- and rod-mediated responses, the data were spatially averaged once $($ ratio $=6)$ and the VERIS noise reduction procedure (NRP) was performed. ${ }^{31}$ Signals were lowpass filtered at $80 \mathrm{~Hz}$ (cones) and $15 \mathrm{~Hz}$ (rods) resulting in better measurable signals for the fitting method without appreciable 
amplitude loss; cone and rod data were fitted over $55 \mathrm{~ms}$ (from 15 to $70 \mathrm{~ms}$ ) and $120 \mathrm{~ms}$ (from 60 to $180 \mathrm{~ms}$ ), respectively, to avoid early and late transients.

We applied the previously described methods ${ }^{21}$ and analysed the results by comparing the averaged central (cen) and peripheral (peri) (CP method) as well as superior (sup) and inferior (inf) (SI method) a- and $\mathrm{t}$-scales. We further analysed the a- and $\mathrm{t}$-scales of each of the 103 and 61 local responses.

Cone-mediated mfERGs were analysed from 13 ARM eyes and from 13 control eyes at baseline and after 1 year. Rod-mediated mfERGs were analysed from 11 ARM eyes and 11 control eyes (ARM subjects 1 and p and two control subjects could not perform the rod mfERG procedure at both times) at baseline and after 1 year.

\section{Psychophysical tests}

Vision function tests were performed monocularly using standardized and recommended procedures at baseline and after 1 year. All subjects underwent the following tests: high contrast distance visual acuity (HC-VA, Bailey-Lovie charts), ${ }^{32}$ contrast sensitivity (PelliRobson), 33,34 colour vision (Lanthony desaturated D-15, desat. D-15) $)^{35,36}$ and central visual fields (10-2 threshold, Humphrey-Zeiss 630, San Leandro, CA, USA). Baseline results have been published in part previously. ${ }^{11}$

\section{Statistical analysis}

Analyses comparing mean and local cone- and rodmediated results were performed using analysis of variance (ANOVA) techniques as computed by the Statistical Package for the Social Sciences (SPSS-11). Repeated measures analyses of variance (ANOVA) within subjects (time, retinal location) and between subjects (group) were conducted.

\section{Results}

\section{Mean cone- and rod-mediated mfERG results}

On average there was a trend of lower mean a-scales for the ARM group compared to the control group for both cone- and rod-mediated responses for the CP and SI methods but this was not significant for either time (Figure 2a). For the mean t-scales significantly delayed latencies $(P \leqslant 0.04)$ were evident for all locations for the rod- but not for the cone-mediated results at both times (Figure 2b). Figure 3 shows the trace arrays of the rodmediated mfERG for the control group and the delayed waveforms for the ARM group at baseline and after 1 year with the averaged overall responses on the right-hand side.
The repeated measures ANOVA for the mean a- and $\mathrm{t}$-scales for the cones showed no significant effect for both methods. There was no significant group effect and no significant interaction between the groups by time and by locations. The rod-mediated mfERG demonstrated a significant group effect with longer latencies for the ARM group compared to the control group for both methods at baseline and after 1 year (CP method: $\mathrm{t}$-scale: $F_{(1,20)}=5.08, P=0.04$; SI method: $\left.F_{(1,20)}=5.6, P=0.03\right)$. However, no significant interactions was found.

\section{Local cone- and rod-mediated mfERG results}

Although there were significantly lower amplitudes for the local cone-mediated responses for the ARM group compared to the control group at baseline $(P<0.01)$ amplitudes were within the normal range after 1 year and this was reflected in a significant group by time interaction (Table 2, Figure 4). A significant group effect demonstrated faster local latencies for the ARM group at baseline and after 1 year $(P=0.03)$ compared to the control group but no group by time interaction was evident here.

There were lower local amplitudes for the rodmediated responses of the ARM group compared to the control group at both times, but these were only significant after 1 year $(P<0.01)$. A group by time interaction revealed that this significant difference was due to higher amplitudes for the control group after 1 year (Table 3, Figure 4). Further the local rod-mediated latencies showed a significant group effect with delayed latencies for the ARM group compared to the control group at both times $(P<0.01)$. No group by time interaction was evident (Table 3 ).

\section{Psychophysical results}

HC-VA, contrast sensitivity and desaturated colour vision (mainly Tritan defect, Table 1) were significantly impaired in the ARM group at baseline compared with the control group $(P \leqslant 0.01)$ but did not change over time, while central visual fields were within the normal range at both times (Table 4). The results of the repeated measures ANOVA show a significant group effect for the HC-VA $\left(F_{(1,24)}=20.1, P<0.01\right)$, the Pelli-Robson $\left(F_{(1,24)}=16.5, P<0.01\right)$ and the desaturated Panel D-15 $\left(F_{(1,23)}=7.3, P \leqslant 0.01\right)$ but no significant group by time effect. Thus, these test results were significantly impaired in the ARM group compared to the control group at both times but showed no further deterioration after 1 year. MS was not significantly different between the groups at baseline or after 1 year. 

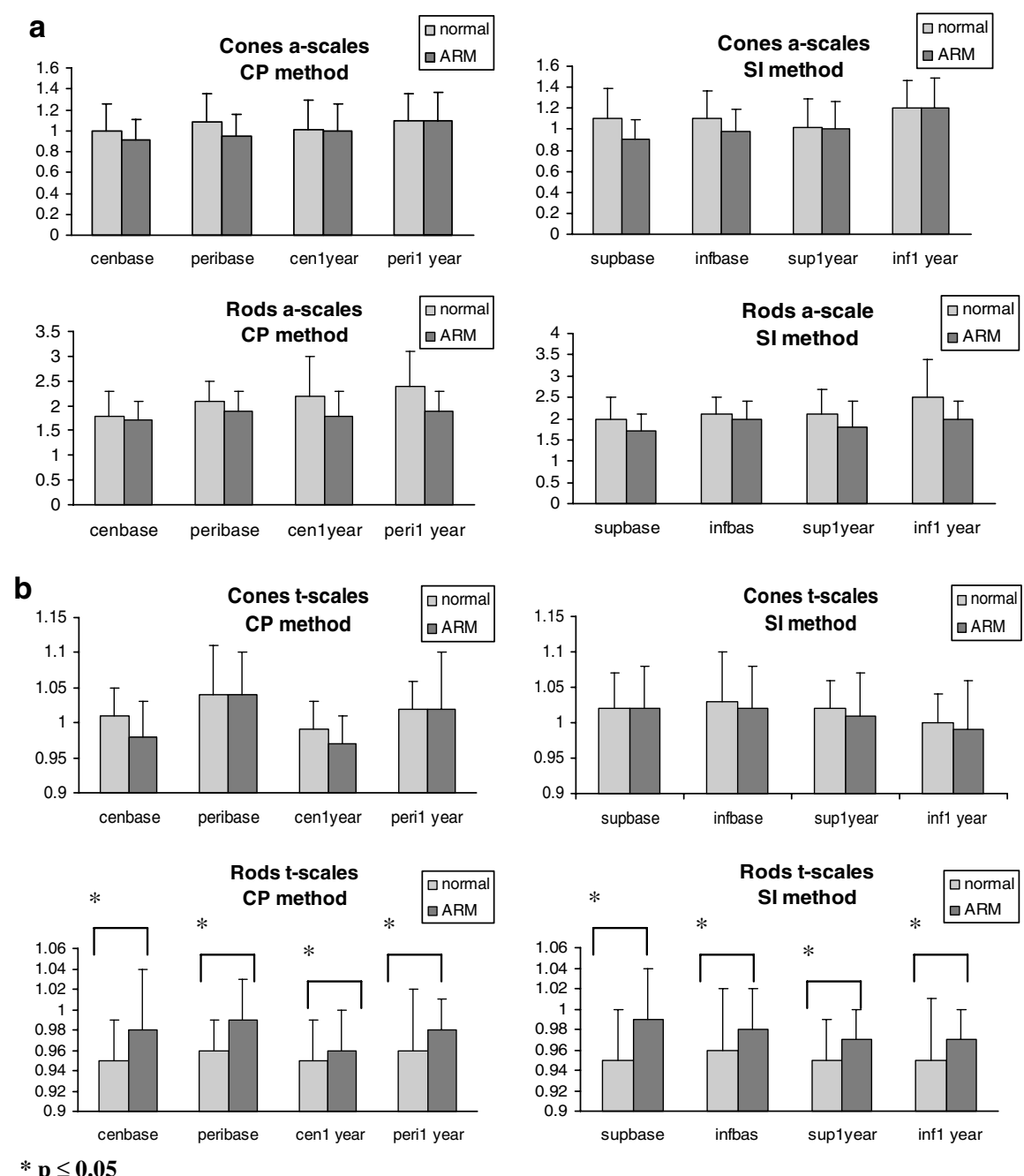

Figure 2 (a) The mean a-ratios (ordinate) for the central and peripheral (CP method) and for the superior and inferior field (SI method) at baseline (cenbase, peribase, supbase, infbase) and after 1 year (cen1year, peri1year, sup1year, inf1year) for the cone- (upper graphs) and rod-mediated (lower graphs) mfERG. Although there were slightly lower mean a-scales for the ARM group compared to the control group this was not statistically significant. (b) The mean t-scale ratios for both methods show significantly delayed rod and slightly faster but not significantly cone-mediated latencies for the ARM group for both methods.

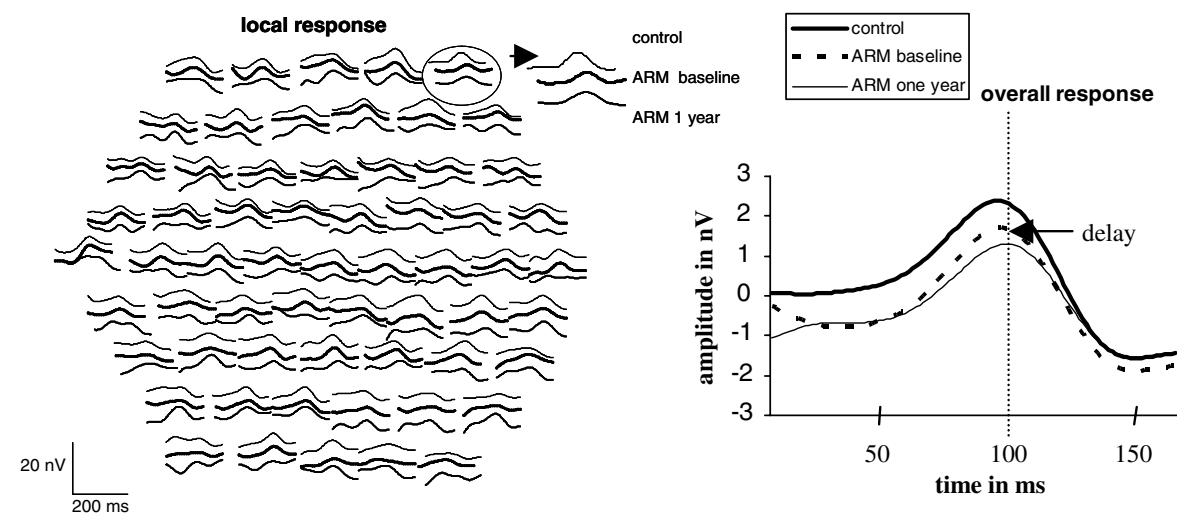

Figure 3 The local responses of the rod-mediated mfERG for the control (upper waveform), the ARM group at baseline (middle waveform) and after 1 year (lower waveform) showing delayed responses for the ARM group. On the right-hand side the overall responses with delayed latencies for the ARM group at baseline and after 1 year compared to the control group are shown. 


\section{Grading results}

We found progressive fundus changes in only two $(18 \%)$ subjects ( $\mathrm{c}$ and $\mathrm{k}$ ) who developed more or new retinal pigment epithelium (RPE) abnormalities (Table 1). Subject $\mathrm{k}$ was graded to a lower AREDS level (LII c3 from LIII b) because of new RPE abnormalities which have

Table 2 Results of the repeated measures analyses of variance for the local cone-mediated mfERG responses

\begin{tabular}{|c|c|}
\hline & Local (103) responses \\
\hline \multicolumn{2}{|l|}{$a$-scales } \\
\hline Group effect & $\begin{array}{l}F_{(1,102)}=36.4 \\
P<0.01^{*} \text { (between ARM and control } \\
\text { group at baseline) }\end{array}$ \\
\hline Group $\times$ time & $\begin{array}{l}F_{(1,102)}=63.8 \\
P<0.01^{*} \text { (between ARM at baseline } \\
\text { and ARM after } 1 \text { year) }\end{array}$ \\
\hline \multicolumn{2}{|l|}{$t$-scales } \\
\hline Group effect & $\begin{array}{l}F_{(1,102)}=5.2 \\
P=0.03^{*} \text { (significantly faster } \\
\text { latencies between ARM and control group } \\
\text { at baseline and after } 1 \text { year) }\end{array}$ \\
\hline Group $\times$ time & $\begin{array}{l}F_{(1,102)}=1.1 \\
P=0.3\end{array}$ \\
\hline
\end{tabular}

*Statistically significant.
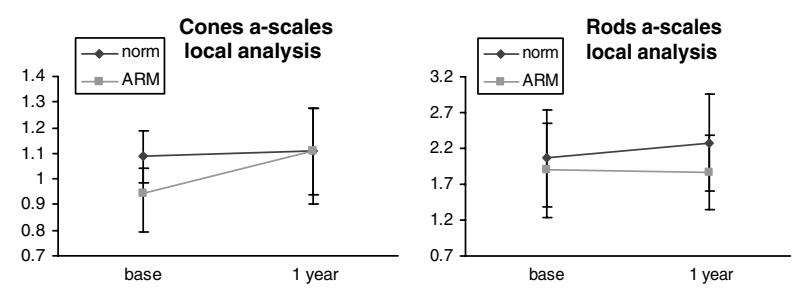

Figure 4 The means and standard deviations of the 103 and 61 hexagons for the a-scales of the local cone-and rod-mediated analysis are shown. A significant group by time interaction for the ARM and control group (norm) was evident. The ARM group demonstrated significantly higher cone-mediated a-scales after 1 year compared to baseline results and thus values comparable to the control group (left graph). Similarily the rodmediated responses of the control group showed a significant increase for the a-scales after 1 year compared to baseline (right graph). been shown to increase the risk of developing late ARM by about three-fold. ${ }^{37}$ None of the control subjects developed early ARM after 1 year.

\section{Discussion}

Our results demonstrate impaired cone- and rod-mediated function at baseline with no further deterioration over a period of 1 year in the early ARM group. By using averaging methods (CP and SI) for the analysis of the mfERG results, a significant delay of the rod-mediated function was evident whereas cone function was within the normal range at both measurement times. Although the local analysis of the cone- and rod-mediated responses showed reduced amplitudes for the ARM group compared to the control group this was not the case after 1 year. However, consistent with the averaging method and our previous study, ${ }^{21}$ we found delayed local rod-meditated and faster local cone-mediated latencies responses for the ARM group. Further, when we used a $P$ value $<0.01$, appropriate to the number of tests we performed, the local rod-mediated latencies together with the conemediated psychophysical measures appeared to detect

Table 3 Results of the repeated measures analyses of variance for the local rod-mediated responses

\begin{tabular}{cl}
\hline & Local (61) responses \\
\hline a-scales & \\
Group effect & $F_{(1,60)}=19.8$ \\
& $P<0.01^{*}$ (between ARM and control \\
& group after 1 year) \\
& $F_{(1,60)}=6.1$ \\
Group $\times$ time & $P=0.02^{*}$ (between control group \\
& at baseline and after 1 year) \\
& \\
t-scales & $F_{(1,60)}=13.6$ \\
Group effect & $P<0.01^{*}$ (between ARM and control \\
& group at both times) \\
Group $\times$ time & $F_{(1,60)}=2.6$ \\
& $P=0.1$ \\
\hline
\end{tabular}

*Statistically significant.

Table 4 Mean results and standard deviations (SD) for the visual acuity (HC-VA), contrast sensitivity (Pelli), desaturated colour vision (desat. D-15) and central visual fields (MS) of the early ARM and control groups at baseline (ARM base, control base) and after 1 year (ARM 1 year, control 1 year)

\begin{tabular}{lcccc}
\hline & ARM base & ARM 1 year & Control base & Control 1 year \\
\hline HC-VA (log Mar) & $0.14( \pm 0.11)^{*}$ & $0.18( \pm 0.11)^{*}$ & $-0.01( \pm 0.13)$ & $0.0( \pm 0.09)$ \\
Pelli (no. of letters) & $32( \pm 3)^{*}$ & $33( \pm 3)^{*}$ & $36( \pm 3)$ & $35( \pm 2)$ \\
desat D-15 (index) & $2.04( \pm 0.7)^{*}$ & $2.4( \pm 0.8)^{*}$ & $1.5( \pm 0.5)$ & $1.8( \pm 0.6)$ \\
MS (dB) & $28( \pm 2)$ & $29( \pm 2)$ & $29( \pm 3)$ & $30( \pm 2)$ \\
\hline
\end{tabular}

${ }^{*} P \leqslant 0.01$ significantly impaired compared to the control group at both times. 
deficits best. Thus, our hypothesis that the rod-mediated mfERG would be the best of the tests we used for detecting functional impairment was not verified in our ARM sample.

The group by time effects showed higher local coneand rod-mediated amplitudes after 1 year. This might be explained by the higher intraindividual variability for amplitudes $^{28}$ than for latencies which has previously been shown for the cone-mediated mfERG. Additionally in our recent study, we showed an amplitude increase for the normal control group by about $28 \%$ over a time period of 1 year but less variability in latencies for the rods. ${ }^{21}$ Another reason for higher amplitudes for the rods after 1 year may be a stray light influence which is thought to be greater in an older population according to Hood et al. ${ }^{20}$ Variation in pupil size and retinal illumination can also have an influence on the mfERG responses. ${ }^{38}$ Chan and Brown ${ }^{38}$ found that foveal mfERG amplitude increased with a slope of $5.24 \mathrm{nV} / \mathrm{mm}^{2}$ of increased pupil area; this would predict an increase of about $120 \mathrm{nV}$ in $\mathrm{mfERG}$ amplitude as pupil size increased for example from 6 to $8 \mathrm{~mm}$ in diameter. However, as our subjects' pupils were maximally dilated at both visits and we do not expect larger pupils after 1 year especially in older subjects, we do not think this was the case in our findings.

We therefore suggest greater reliance on latencies in detecting early changes than on amplitudes as they seem to be more robust. Delayed latencies in the conemediated mfERG have been shown to be early indicators in $\mathrm{ARM}^{14}$ and in other diseases such as diabetes and hereditary retinal conditions. ${ }^{29,30,39}$ Han et $a l^{30}$ demonstrated that new diabetic retinopathy was predicted by delayed latencies in an area without diabetic retinopathy a year before diabetic retinopathy developed. Whether local faster cone-mediated latencies for the ARM group compared to the control group reflect altered synaptic transmission and beginning pathology or not still needs to be investigated, but we did find delayed rod-mediated latencies in the local and averaging analysis methods at both times.

Curcio et al ${ }^{40,41}$ demonstrated a selective vulnerability of parafoveal rods over cones in early ARM and a progressive rod loss of $30 \%$ throughout the lifespan. The reason that we could not show any significant change in rod-mediated function over time might be because the loss is very slow, affecting only about 2 rods $/ \mathrm{mm}^{2}$ per year. ${ }^{41}$ Curcio et al ${ }^{40}$ further demonstrated that there were no gaps in the retina as the inner segments of the remaining rods expanded. It is possible that reduced rod function is compensated by the remaining rods at the beginning of the ARM disease process.

Fundus changes also did not progress in most of our subjects over this time period but slow progression in
ARM has been described in other studies. ${ }^{37,42}$ The Rotterdam study ${ }^{37}$ has demonstrated a slow but constant progression in ARM severity with age and time following a distinct, stage by stage course. After 2 years, the risk for developing the next stage of ARM was between 5 and 30\%. Interestingly, this was lowest (5\%) for the most advanced stage but increased to about $40 \%$ after 6.5 years, whereas less-advanced stages showed a progression rate to the next stage of less than $10 \%$ after 6.5 years. Given the slow progression rate, the short follow-up of our subjects and the fact that most of our subjects had more progressed fundus changes to begin with (Table 1) our functional and morphological results seem reasonable.

Our study further is in accordance with findings of several other studies showing that colour vision ${ }^{3,4,6}$ and contrast sensitivity ${ }^{5,43}$ are sensitive in detecting early impairment of cone function subjectively in ARM. Whether follow-up with the rod-mediated mfERG over a longer period may indicate a significantly faster and greater rod-mediated impairment or not, as has been demonstrated with psychophysical tests, ${ }^{16,44}$ still needs to be investigated. In future monitoring rod function might become an important issue in the treatment of ARM. Mohand-Said et $a l^{45}$ found protective effects of transplanted rods on host cones in animal experiments. It has been hypothesised that this might help the survival of cones and that postponing or blocking death of rod cells, for example by the use of pharmacological agents, might provide future treatment options. ${ }^{46,47}$ However, given that the rod-mediated mfERG is a time-consuming procedure, ${ }^{20,21}$ shorter and less-demanding protocols for assessing rod function would be more useful in the clinical setting. Further research is needed to establish if it is necessary to test close to the absolute threshold and if different colour or luminance backgrounds suppress or minimize cone function.

\section{Acknowledgements}

We thank Dion Scott for helping to develop the statfit analysis using Matlab (Mathworks, Natick, MA, USA) and Andrew Carkeet for helpful statistical assistance.

\section{References}

1 Brown B, Tobin C, Roche N, Wolanowski A. Cone adaptation in age-related maculopathy. Am J Optom Physiol Opt 1986; 63: 450-454.

2 Sunness J, Johnson M, Massof R, Marcus S. Retinal sensitivity over drusen and nondrusen areas. Arch Ophthalmol 1988; 106: 1081-1084.

3 Midena E, Degli A, Blarzino M, Valenti M, Segato T. Macular function impairment in eyes with early age-related 
macular degeneration. Invest Ophthalmol Vis Sci 1997; 38: 469-477.

4 Eisner A, Stoumbos V, Klein M, Fleming A. Relation between fundus appearance and function. Eyes whose fellow eye has exudative age-related macular degeneration. Invest Ophthalmol Vis Sci 1991; 32: 8-20.

5 Kleiner R, Enger C, Alexander M, Fine S. Contrast sensitivity in age-related macular degeneration. Arch Ophthalmol 1988; 106: 55-57.

6 Collins M. Pre-age related maculopathy and the desaturated D-15 colour vision test. Clin Exp Opt 1986; 69: 223-227.

7 Sunness J, Massof R, Johnson M, Bressler N, Bressler S, Fine F. Diminished foveal sensitivity may predict the development of advanced age-related macular degeneration. Ophthalmology 1988; 96: 375-381.

8 Owsley C, Jackson G, Cideciyan A, Huang Y, Fine S, Ho A et al. Psychophysical evidence for rod vulnerability in age-related macular degeneration. Invest Ophthalmol Vis Sci 2000; 41: 267-273.

9 Curcio C, Medeiros N, Millican L. Photoreceptor loss in age-related macular degeneration. Invest Ophthalmol Vis Sci 1996; 37: 1236-1249.

10 Jackson G, Owsley C, Curcio C. Photoreceptor degeneration and dysfunction in aging and age-related maculopathy. Ageing Res Rev 2002; 1: 381-396.

11 Feigl B, Brown B, Lovie-Kitchin J, Swann P. Cone-mediated multifocal electroretinogram in early age-related maculopathy and its relationships with subjective macular function tests. Curr Eye Res, in press.

12 Phipps J, Guymer R, Vingrys A. Loss of cone function in age-related maculopathy. Invest Ophthalmol Vis Sci 2003; 44: 2277-2283.

13 Falsini B, Fadda A, Iarossi G, Piccardi M, Canu D, MInnella A et al. Retinal sensitiviy to flicker modulation: reduced by early age-related maculopathy. Invest Ophthalmol Vis Sci 2003; 41: 1498-1506.

14 Gerth C, Hause D, Delahunt P, Morse L, Werner J. Assessment of multifocal electroretinogram abnormalities and their relation to morphologic characteristics with large drusen. Arch Ophthalmol 2003; 121: 1404-1414.

$15 \mathrm{Li} \mathrm{J}$, Tso M, Lam T. Reduced amplitude and delayed latency in foveal response of multifocal electroretinogram in early age related macular degeneration. Br J Ophthalmol 2001; 85: 287-290.

16 Scholl H, Bellmann C, Dandekar S, Bird A, Fitzke F. Photopic and scotopic fine matrix mapping of retinal areas of increased fundus autofluorescence in patients with age-related maculopathy. Invest Ophthalmol Vis Sci 2004; 45: 574-583.

17 Jackson G, Owsley C, Cordle E, Finley C. Aging and scotopic sensitivity. Vision Res 1998; 38: 3655-3662.

18 Curcio C, Owsley C, Jackson G. Spare the rods, save the cones in aging and age-related maculopathy. Invest Ophthalmol Vis Sci 2000; 41: 2015-2018.

19 Owsley C, Jackson G, White M, Edwards D. Delays in rodmediated dark-adaptation in early age-related maculopathy. Ophthalmology 2001; 108: 1196-1202.

20 Hood D, Wladis E, Shady S, Holopigian K, Li J, Seiple W. Multifocal rod electroretinograms. Invest Ophthalmol Vis Sci 1998; 39: 1152-1161.

21 Feigl B, Brown B, Lovie-Kitchin J, Swann P. Cone-and rod-mediated $\mathrm{mfERG}$ in early age-related maculopathy. Eye, advance online publication 30 July 2004.
22 The Age-Related Eye Disease Study Research Group. The age-related eye disease study system for classifying cataracts from photographs: AREDS report number 4. Am J Ophthalmol 2001; 131: 167-175.

23 The Age-Related Eye Disease Study Research Group. The age-related eye disease study system for classifying agerelated macular degeneration from stereoscopic color fundus photographs: the age-related eye disease study report number 6. Am J Ophthalmol 2001; 132: 668-681.

24 Johnson P, Lewis G, Talaga K, Brown M, Kappel P, Fisher S et al. Drusen-associated degeneration in the retina. Invest Ophthalmol Vis Sci 2003; 44: 4481-4488.

25 Hood D, Li J. A technique for measuring individual multifocal ERG records. Noninvasive Assess Visual System: Trends Opt Photon 1997; 11: 33-41.

26 Holopigian K, Seiple W, Greenstein V, Hood D, Carr R. Local cone and rod system function in patients with retinitis pigmentosa. Invest Ophthalmol Vis Sci 2001; 42: 779-788.

27 Holopigian K, Seiple W, Greenstein V, Hood D, Carr R. Local cone and rod system function in progressive cone dystrophy. Invest Ophthalmol Vis Sci 2002; 43: 2364-2373.

28 Seiple W, Vajaranant T, Szlyk J, Clemens C, Holopigian K, Paliga J et al. Multifocal electroretinography as a function of age: The importance of normative values for older adults. Invest Ophthalmol Vis Sci 2003; 44: 1783-1792.

29 Fortune B, Schneck M, Adams A. Multifocal electroretinogram delays reveal local retinal dysfunction in early diabetic retinopathy. Invest Ophthalmol Vis Sci 1999; 40: 2638-2651.

30 Han Y, Bearse MJ, Schneck M, Barez S, Jacobson C, Adams A. Multifocal electroretinogram delays predict sites of subsequent diabetic retinopathy. Invest Ophthalmol Vis Sci 2004; 45: 948-954.

31 Sutter E, Tran D. The field topography of ERG components in man - I. the photopic luminance response. Vision Res 1992; 32: 433-446.

32 Bailey I, Lovie J. New design principles for visual acuity letter charts. Am J Optom Physiol Opt 1976; 53: 740-745.

33 Pelli D, Robson J, Wilkins A. The design of a new letter chart for measuring contrast sensitivity. Clin Vis Sci 1988; 2: 187-199.

34 Elliott D, Whitaker D, Bonette L. Differences in the legibility of letters at contrast threshold using the Pelli-Robson chart. Ophthal Physiol Opt 1990; 10: 323-326.

35 Committee on Vision AoBaSS, National Research Council. Procedures for Testing Color Vision: Report of Working Group 41. National Academy Press: Washington, 1981, pp 57-60.

36 Lanthony P. The desaturated panel D-15. Doc Ophthalmol 1978; 46: 185-189.

37 van Leeuwen R, Klaver C, Vingerling J, Hofman A, de Jong P. The risk and natural course of age-related maculopathy. Follow-up at $61 / 2$ years in the Rotterdam study. Arch Ophthalmol 2003; 121: 519-526.

38 Chan $\mathrm{H}$, Brown B. Investigation of retinitis pigmentosa using the multifocal electroretinogram. Ophthal Physiol Opt 1998; 18: 335-350.

39 Hood D, Holopigian K, Greenstein V, Seiple W, Li J, Sutter E et al. Assessment of local retinal function in patients with retinitis pigmentosa using the multi-focal ERG technique. Vision Res 1998; 38: 163-179.

40 Curcio C, Millican L, Allen K, Kalina R. Aging of the human photoreceptor mosaic: evidence for selective vulnerability of rods in central retina. Invest Ophthalmol Vis Sci 1993; 34: 3278-3296. 
41 Curcio C. Photoreceptor topography in aging and age-related maculopathy. Eye 2001; 15: 376-383.

42 Klein R, Klein B, Tomany S, Meuer S, Huang G. Ten-years incidence and progression of age-related maculopathy: The Beaver Dam Eye study. Ophthalmology 2002; 109: 1767-1779.

43 Brown B, Lovie-Kitchin J. Contrast sensitivity in central and paracentral retina in age-related maculopathy. Clin Exp Opt 1987; 70: 145-148.

44 Jackson G, Owsley C. Scotopic sensitivity during adulthood. Vision Res 2000; 40: 2467-2473.
45 Mohand-Said S, Hicks D, Leveillard T, Picaud S, Porto F, Sahel JA. Rod-cone interactions: developmental and clinical significance. Prog Retin Eye Res 2001; 20: 451-467.

46 Liu C, Li Y, Peng M, Laties A, Wen R. Activation of caspase3 in the retina of transgenetic rats with the rhodopsin mutation s334ter during photoreceptor degeneration. J Neurosci 1999; 19: 4778-4785.

47 LaVail M, Unoki K, Yasumura D, Matthes M, Yancopoulos G, Steinberg R. Multiple growth factors, cytokines and neurotrophins rescue photoreceptors from damaging effects of constant light. Proc Natl Acad Sci USA 1992; 89: 11249-11253. 